\title{
On the significance of periodic signals in noise analysis of GPS station coordinates time series
}

\author{
Janusz Bogusz ${ }^{1} \cdot$ Anna Klos ${ }^{1}$
}

Received: 16 March 2015/Accepted: 15 July 2015/Published online: 28 July 2015

(C) The Author(s) 2015. This article is published with open access at Springerlink.com

\begin{abstract}
Each of the GPS-derived time series consists of the deterministic (functional) and stochastic part. We propose that the deterministic part includes all periodicities from 1st to 9th harmonics of residual Chandler, tropical and draconitic periods and compare it with commonly used calculations of the annual and semi-annual tropical curve. Then, we address the issues of whether all residual periodicities, as proposed here, need to be taken into consideration when performing noise analysis. We use the position time series from 180 International GNSS Service stations obtained at the Jet Propulsion Laboratory using the GIPSY-OASIS software in a Precise Point Positioning mode. The longest series has 22.1 years of GPS daily solutions. The spectral indices range from -0.12 to -0.92 , while the median values of "global" spectral indices are equal to: $-0.41 \pm 0.15,-0.38 \pm 0.12$ and $-0.33 \pm 0.18$ for North, East and Up components, respectively. All nonmodelled geophysical processes or non-included artificial effects in time series lead to an underestimation of errors of velocities, but also to changes in the velocity values themselves. The proposed assumption of seasonals subtraction caused the Akaike information criterion values to show a decrease in the median value of $30 \%$, which in fact means that all the seasonals mentioned here must be taken into account when analyzing noises. Finally, we noticed that there are some of the GPS stations that improved their velocity uncertainty even of $56 \%$.
\end{abstract}

Anna Klos

anna.klos@wat.edu.pl

1 Faculty of Civil Engineering and Geodesy, Military University of Technology, Kaliskiego St. 2, 00-908 Warsaw, Poland
Keywords Seasonal signals - Noise analysis - PPP solution · GPS · IGS

\section{Introduction}

Scientists who study the solid earth are increasingly using the GPS system. It offers a wide range of applications that allow the determination of not only long-term changes, like tectonic or glacial isostatic adjustment studies with daily or weekly solutions, but also to investigate either long-term geophysical signals or sub-daily changes, including seismic and volcanic deformations, or ice sheet flow. When creating a subsequent kinematic reference frame, the most important issue is to ensure its optimum definition and stability or reliability as a function of time. While the origin and scale of reference frames with their physical characteristics are the main parameters used for numerous applications related to the earth sciences, the knowledge on earth's orientation and its changes over time is necessary to ensure the continuity of the observation of earth rotation. Any, even a small change in the aforementioned is transferred to all geophysical results based on the International Terrestrial Reference Frame (ITRF), for example the average sea level.

For all those tasks mentioned, the proper determination of velocity of a permanent station and its uncertainties is essential. Therefore, the knowledge of seasonal changes is also crucial. Among all the oscillations with a period close to 1 year, these should be distinguished: a tropical year (365.2421 days), i.e., time within when the earth returns to the same position with respect to the sun, and a draconitic year ( $\sim 351$ days), i.e., time within when the constellation of GPS satellites return to the same position with respect to the sun. 
The first periodic variations with a period of tropical year may reflect a common physical basis, such as seasonal mass redistribution (van Dam et al. 1994; Blewitt and Lavallée 2002; Wu et al. 2006), or mismodelling in short periods (Dong et al. 2002, Penna and Stewart 2003). However, all these seasonal variations can be modelled by the set of the harmonic functions as shown in Amiri-Simkooei et al. (2007). Moreover, they showed that the more the harmonic components of seasonal oscillation assumed to be present in GPS time series, the closer the stochastic part is to white noise. Bos et al. (2010) noticed that under equal noise conditions, the worst accuracy of linear velocity was obtained from the time series biased with seasonal signals. They also stated that omitting noise properties causes significant variations in the parameters estimated from the GPS data.

The second periodic variation with frequency of a draconitic year is an effect of a systematic, technique-related error associated with satellite orbits. It has already been discovered in GPS time series by several researchers. Agnew and Larsson (2007) used broadcast ephemerides of all GPS satellites operating between 1996 and 2006 to find orbital repeat times. They stated that for daily sampling rates, this period will alias to a frequency of $1.04333 \mathrm{cpy}$. Ray et al. (2008) examined spectra of more than 160 IGS permanent stations. Having the length of them equal to 200 weeks, they analyzed the 6th harmonics. They calculated $1.040 \pm 0.008$ cpy (351.2 year) frequency with a linear overtone assumed and called it the "GPS year." They found no confirmation of such harmonics either in VLBI or SLR, or in geophysical fluids. Collilieux et al. (2007) investigated height residual time series of VLBI, SLR and GPS submitted to the ITRF2005. Apart from significant power near 1 and 2 cpy, they also found 3.12 and $4.16 \mathrm{cpy}$, being detectable in the individual time series. Santamaría-Gómez et al. (2011) analyzed time series from a homogeneously reprocessed solution of 275 globally distributed stations in terms of noise content and velocity uncertainty assessment. Before velocity estimation, they subtracted annual and semi-annual signals from the data of a minimum length of 2.5 years. They verified the presence of the residual periodicities by the nonlinear iterative least squares (LS) method and found substantial amplitudes in the periods from 1 st to 4 th harmonics, while very little significance from the 5th harmonic onward. Amiri-Simkooei (2013) analyzed JPL data processed at the GPS Analysis Center to obtain a significant signal with a period of $351.6 \pm 0.2$ days and its higher harmonics in North, East and Up time series. He used the least squares harmonic estimation (LS-HE) for detecting and including a set of harmonic functions for the periodicities in the considered time series. He obtained the variation range of this periodic pattern of about $3.0,3.2$ and $6.5 \mathrm{~mm}$ amplitude for all three components, respectively, and found the white and flicker noise to be prevailing in the position time series with a relatively small contribution of random walk noise. Griffiths and Ray (2013) discovered harmonic signals (draconitic and overtones up to at least sixth) in the power spectra of nearly all IGS products by reconsidering how errors in the conventional model fit into GPS orbit and EOP (Earth Orientation Parameters) products. They suggested two main driving mechanisms: bias due to mismodelling of orbit dynamics and local multipath effects. RodriguezSolano et al. (2012) investigated global network (CODE solutions) and explained the 6th overtone of the draconitic year in GPS station positions estimates (at the mm level) by the earth radiation pressure, arising from wrong modelling over large regions.

Using GPS in combination with some other techniques, one can successfully isolate the cause of the polar motion wobble. Gross et al. (2004) using GPS data collected between 1997 and 2000 predicted how the position of the North Pole would vary given changes in earth's mass distribution and then compared the predicted wobble to the direct observations of the axis made during the same period by satellites and very long baseline interferometry. Kouba (2005) used polar motion combined series produced by the International GPS Service (IGS) using only GPS data and compared with the combined excitations of atmosphere and oceans at all intervals, showing high overall correlation of 0.8-0.9. The effect of mismodelling of the Chandler period and its overtones can be observed in GPS-derived coordinates. Collilieux et al. (2007) found a wide range of frequencies detected between 0.75 and 0.9 cpy in GPS, VLBI and SLR height residuals from ITRF2005 solution. We would expect a signal at the Chandler period to be present at some stations, where the change in sea surface heights loads the crust, causing a loading signal at the Chandler period that would be largest at island and coastal sites (Richard Gross, personal communication). The oceanic pole tide is the response of the oceans to the wobble of the underlying solid earth. This also introduces a small signal at the Chandler period in sea surface height measurements (Desai 2005).

\section{Time series}

We used here the position time series obtained at the Jet Propulsion Laboratory (JPL) using the GIPSY-OASIS software in a Precise Point Positioning (PPP) mode, available at the ftp://sideshow.jpl.nasa.gov/. The details concerning processing of GNSS observations can be found at https://gipsy-oasis.jpl.nasa.gov. At the pre-analysis stage, the outliers and offsets were detected using median absolute deviation (MAD) criterion and sequential $t$ test 
analysis of regime shifts (STARS) algorithm (Rodionov and Overland 2005). Detecting discontinuities with STARS is divided into two steps: In the first one, it is checked whether the distance of each point from the local mean is significant according to a $t$ test. In second one, the jump candidates are accepted using inequality with some empirical parameters. A more detailed description about implementing STARS in geodetic time series can be found in Bruni et al. (2014). Although this is self-acting procedure, we used it only for indicating possible steps, after that we removed them manually as recommended in Gazeaux et al. (2013) or described in Williams (2003a). The missing data (gaps no longer than 40 days) were filled with a white noise. Even though adding white noise will weaken the real signals, such an interpolation was done, because maximum-likelihood estimation (MLE) gives more realistic results when performed on continuous data (Bos et al. 2008).

To separate the tropical and draconitic curves of 365.2 and 351.6 days with spectral analysis, one will need at least 25.5 years of continuous data. This length comes from the Rayleigh criterion-minimum length of series required to resolve two constituents (Godin 1972). The sinusoidal model of the least squares estimation which determines all periodicities simultaneously will allow us to determine the amplitudes and phases of both the tropical and the draconitic years and its harmonics for data shorter than 25.5 years, being completely sure of their presence in the data (Tom Herring, personal communication). However, we introduced here a threshold of 12.8 years in the length of the data in order to be sure that the 2nd tropical and draconitic harmonics were resolved in spectral estimation, which resulted in the number of globally distributed stations limited to 180 out of the whole IGS network (Fig. 1). The longest series contained 22.1 years of GPS daily solutions.
It is widely acknowledged that any periodic signal consists of the principal frequency as well as its higher harmonics. Due to this, we assumed that the IGS time series contains both the deterministic part by means of functional model that includes trend and seasonality as well as background noise (stochastic part). Additionally, we incorporated oscillations of 13.66 days corresponding to Mf body tide (Melchior 1983), 14.6 days reported in Amiri-Simkooei (2013), 14.19 and 14.76 days which are the GPS aliasing periods pointed out in Griffiths and Ray (2013), into our functional model. The final form of the equation describing the time series is:

$$
\begin{aligned}
x(t)= & x_{0}+v_{x} \cdot t+A^{13.66} \cdot \sin \left(\omega^{13.66} \cdot t+\phi^{13.66}\right) \\
& +A^{14.6} \cdot \sin \left(\omega^{14.6} \cdot t+\phi^{14.6}\right) \\
& +A^{14.19} \cdot \sin \left(\omega^{14.19} \cdot t+\phi^{14.19}\right) \\
& +A^{14.76} \cdot \sin \left(\omega^{14.76} \cdot t+\phi^{14.76}\right) \\
& +\sum_{i=1}^{9}\left[A_{i}^{\mathrm{CH}} \cdot \sin \left(\omega_{i}^{\mathrm{CH}} \cdot t+\phi_{i}^{\mathrm{CH}}\right)\right] \\
& +\sum_{i=1}^{9}\left[A_{i}^{T} \cdot \sin \left(\omega_{i}^{\mathrm{T}} \cdot t+\phi_{i}^{\mathrm{T}}\right)\right] \\
& +\sum_{i=1}^{9}\left[A_{i}^{\mathrm{D}} \cdot \sin \left(\omega_{i}^{\mathrm{D}} \cdot t+\phi_{i}^{\mathrm{D}}\right)\right]+\varepsilon_{x}(t)
\end{aligned}
$$

where the superscripts $C H, T$ and $D$ denote Chandler, tropical and draconitic oscillations, respectively. At the next stage of the analysis, this equation is linearized by introducing in-phase $(A S)$ and out-of-phase $(A C)$ terms, providing LS-based estimation of the unknowns:

$$
\begin{aligned}
A \cdot \sin (\omega \cdot t+\varphi) & =A \cdot \cos \varphi \cdot \sin (\omega \cdot t)+A \cdot \sin \varphi \cdot \cos (\omega \cdot t) \\
& =\mathrm{AS} \cdot \sin (\omega \cdot t)+\mathrm{AC} \cdot \cos (\omega \cdot t)
\end{aligned}
$$

Fig. 1 The 180 IGS stations analyzed. Different colors dots indicate various lengths of the time series in years

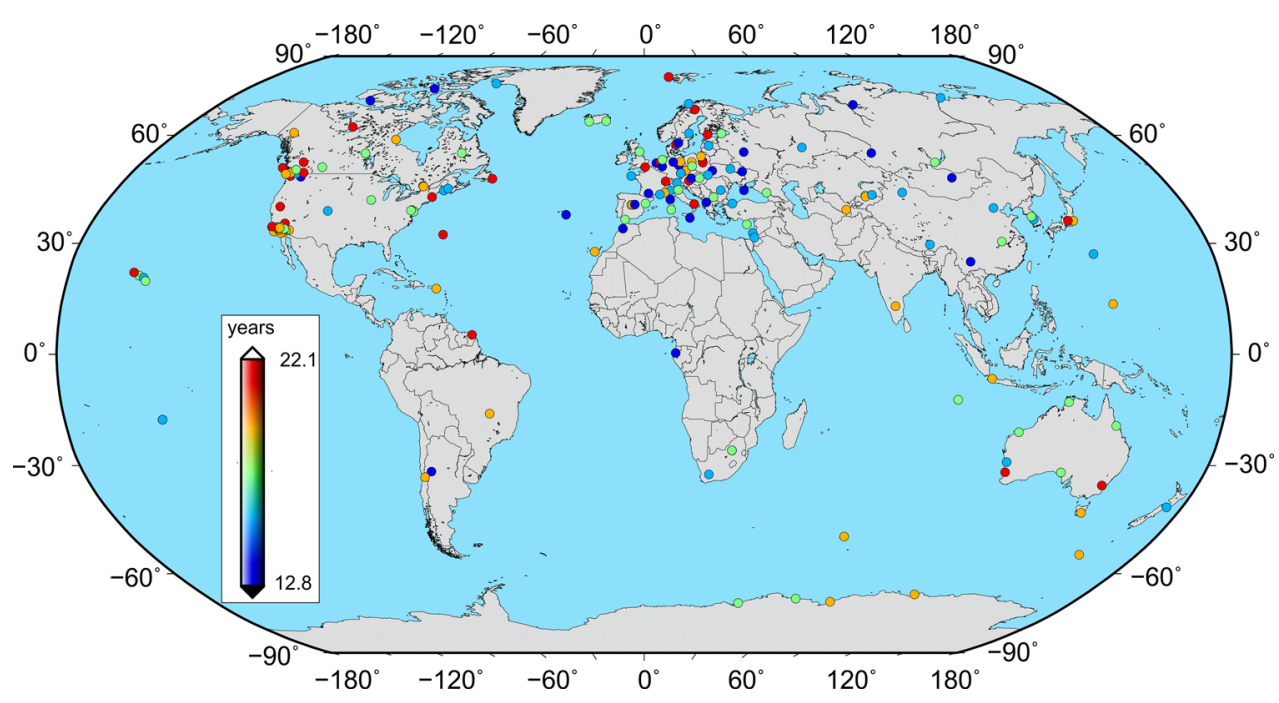


where $\mathrm{AS}=A \cdot \cos \varphi$ and $\mathrm{AC}=A \cdot \sin \varphi . \quad$ In this research, we quantified overtones of basic oscillation up to 9th, which will be proven to be significant in the next chapter. We assumed that the Chandler period is equal to 433.0 days and the draconitic period is equal to 351.6 days following Amiri-Simkooei (2013).

The deterministic part (trend plus seasonal signals) was modelled with the weighted least squares method:

$\mathbf{y}=\left[\mathbf{A}^{\mathrm{T}} \mathbf{C}_{\mathbf{x}}^{-\mathbf{1}} \mathbf{A}\right]^{-\mathbf{1}} \mathbf{A}^{\mathrm{T}} \mathbf{C}_{\mathbf{x}}^{-1} \mathbf{x}$

where $\mathbf{y}$ is the $70 \times 1$ vector of unknowns: interception, linear trend as well as in-phase and out-of-phase seasonal terms combining residual Chandler oscillation, the tropical annual curve and terms with draconitic period up to their 9th harmonic with fortnightly and quasi-fortnightly oscillations, $\mathbf{x}$ is the $n \times 1$ vector of observations $n-$ number of data in North, East or Up components), $\mathbf{C}_{\mathbf{x}}$ is the weight matrix, containing RMSs, and $\mathbf{A}$ is the $n \times 70$ matrix of coefficients. The covariance matrix is then defined as follows:

$\mathbf{C}_{\mathbf{y}}=\left[\mathbf{A}^{\mathrm{T}} \mathbf{C}_{\mathbf{x}}^{-\mathbf{1}} \mathbf{A}\right]^{-\mathbf{1}}$

and contains uncertainties of the unknowns.

\section{Periodicities in IGS data}

Periodic signals will naturally make the data appear to be autocorrelated in the same way that a linear trend would if it is not properly removed. Davis et al.'s (2012) research on seasonal signals in GNSS and GRACE basically attempts to prove that the flicker noise is simply the non-periodic part of the seasonal signal. While it is a reasonable argument, it would mean that the flicker noise would be visible in the spectrum for periods longer than 1 year, which it clearly is. They concluded that noise in the GNSS time series reported by others may be associated with neglecting the stochastic component of the seasonal signal. On the basis of that, the more the seasonal components is assumed in the deterministic model, the less the autocorrelated are the residuals. We propose here that the deterministic part includes all periodicities from 1st to 9th harmonics of residual Chandler, tropical and draconitic periods and compare it with commonly used calculations of the annual and semi-annual tropical curve. Figure 2 (top) presents the daily changes from KUNM station (Kunming, China) with deterministic model containing harmonics proposed here fitted with the weighted least squares approach. The residua are given in bottom panel. Figure 3 presents an exemplary Lomb-Scargle periodogram (Lomb 1976; Scargle 1982) of Up component from the KUNM (Kunming, China) permanent station. The KUNM series is 15 years long, which

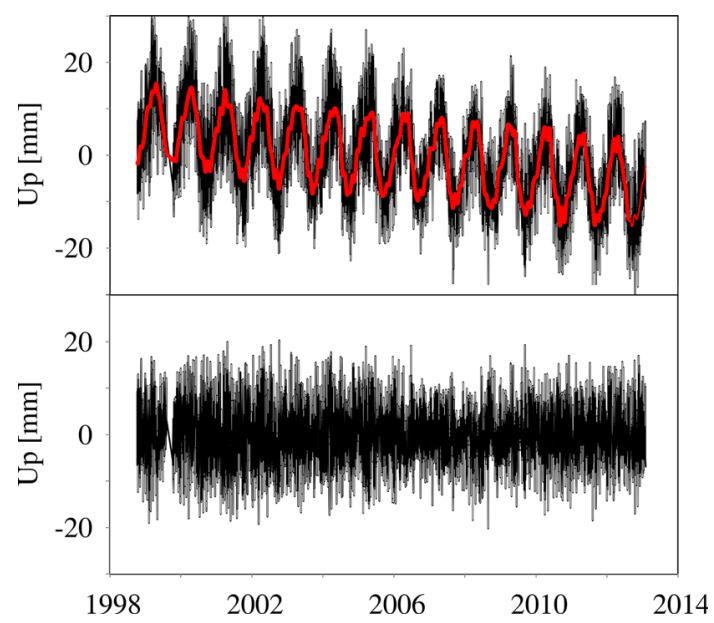

Fig. 2 Fifteen-year time series of Up component (black) given in (mm) from KUNM (Kunming, China). Top panel contains original time series along with the deterministic model ( $r e d$ ) consisting of the best fitted straight line plus all considered sinusoids up to 9th harmonic. Bottom panel contains residuals of the deterministic model

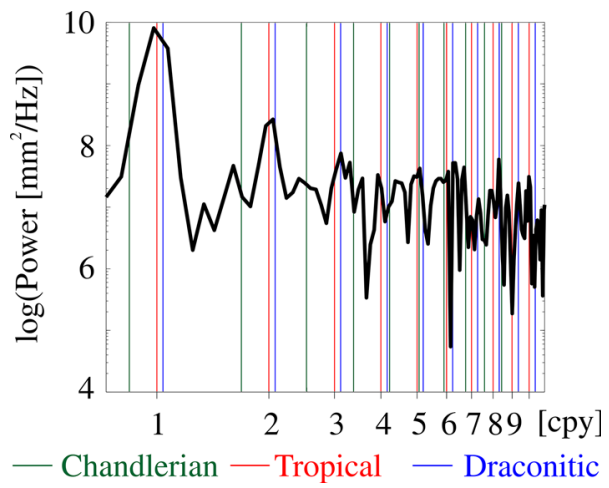

Fig. 3 Lomb-Scargle periodogram in a log-log scale for Up component of station KUNM (Kunming, China). Vertical lines denote Chandlerian, tropical and draconitic harmonics. The horizontal axis was limited to include periods $1-10 \mathrm{cpy}$

means that all oscillations starting from the 2 nd harmonics of tropical and draconitic periods and higher ones can be reliably separated with spectral analysis. Here, the 2nd harmonics are equal to 4.0 and $4.1 \mathrm{~mm}$ for tropical and draconitic year. Significant peak is noticed for 3rd harmonic of draconitic year (3.12 cpy) with the amplitude of $2.7 \mathrm{~mm}$.

The dominant oscillations for the IGS stations were found in the stacked spectra (Fig. 4) for frequencies close to $1 \mathrm{cpy}$, though no conclusions are to be drawn about the complexity of the spectrum. An evident comb of 2 cpy (2nd tropical and draconitic harmonics) was noticed with the predominating tropical oscillation. Although the tropical year prevails in the 1 st and 2 nd harmonics, the draconitic oscillations overweigh others for $3 \mathrm{rd}$, 4th and up to the 10th. Then, again prominent peaks were noticed in 

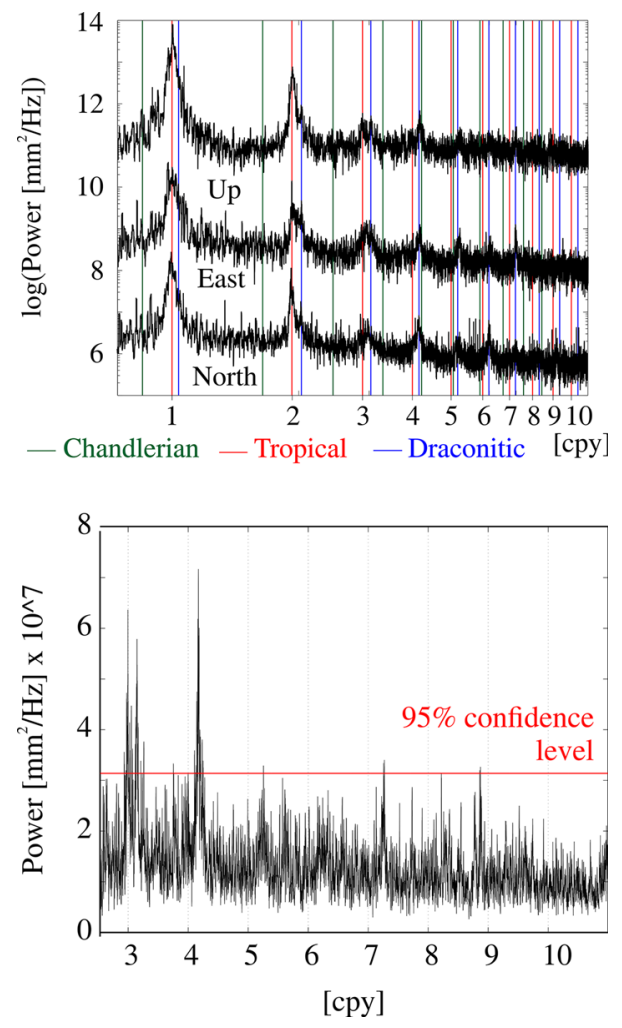

Fig. 4 Stacked spectra averaged with 0.05 cpy window in a log-log scale of North, East and Up components from 180 globally distributed IGS stations (top). The vertical lines denote Chandlerian, tropical and draconitic harmonics. The PSD's of Up and East were artificially shifted in a vertical direction for better interpretation purposes. On bottom the stacked spectra of Up component are given from 3 to 10 cpy with $95 \%$ significance level in a linear scale, for which significant peaks are easy to notice

draconitics around 3-7 cpy, or as it is for North component, where the 9th draconitic harmonic is still significant (9.35 cpy, $3.1 \mathrm{~mm}$ ). In addition, the following is noteworthy: extremely high peaks for 6th harmonic of draconitic oscillation for North $(6.26 \mathrm{cpy}, 1.6 \mathrm{~mm})$, 7th draconitic for East (7.27 cpy, $1.7 \mathrm{~mm})$, whereas tropical oscillations can be noticed only for first three harmonics (up to $3 \mathrm{cpy}$ ). In the Up component, no prominent oscillations were found for periods lower than 5 cpy. The stacked spectra reveal nothing about Chandlerian oscillations, though they are seen up to 9th harmonics (7.59 cpy) with the amplitude of up to $1 \mathrm{~mm}$ for several of IGS stations.

The largest tropical amplitude (determined using LSE) is $8.5 \pm 0.3$ at station KUNM (Kunming, China) in Up component (1st harmonic), while the largest draconitic amplitude is $2.8 \pm 0.2$ at station FAIR (Fairbanks, USA) in Up component (1st harmonic). As expected, all stations with the highest Chandlerian amplitudes (1st harmonic) are located at the Pacific coasts. They are HOLP (Hollydale, USA-1.3 $\pm 0.1 \mathrm{~mm}$ amplitude in Up direction) and UCLU (Ucluelet, Canada- $1.1 \pm 0.1 \mathrm{~mm}$ amplitude in

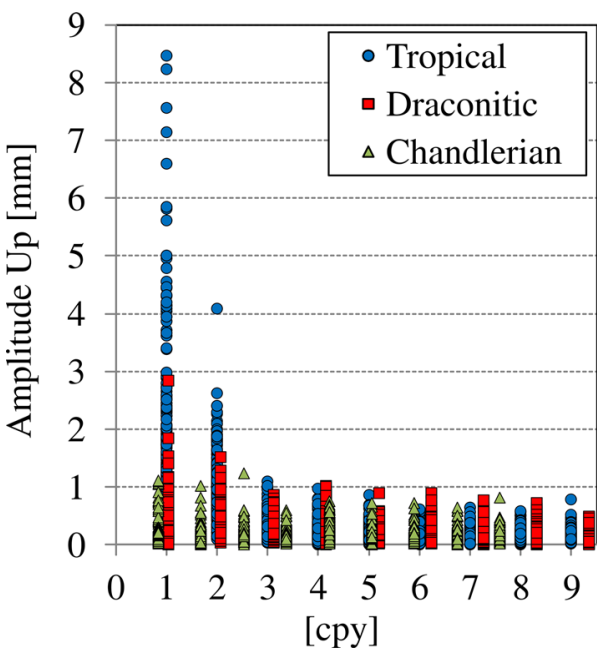

Fig. 5 Amplitudes of tropical, draconitic and Chandlerian residual oscillations in Up component for IGS stations determined using LSE $(\mathrm{mm})$. Tropical amplitudes are presented in blue, draconitic in red and Chandlerian in green

Up). However, these numbers are greater than expected from the ocean pole tide loading model by a factor of about 3 (Shailen Desai, personal communication). Details concerning magnitudes of oscillations and their overtones are presented in Fig. 5.

The largest oscillations were observed in the periods corresponding to 1 tropical year, but their presence due to mismodelling in the short periods (Penna et al. 2007; King et al. 2008; Bogusz and Figurski 2012) is what has to be kept in mind. Moreover, Tregoning and Watson (2009) remarked that omitting diurnal and semi-diurnal atmospheric tidal effects can introduce an artificial signal close to the draconitic period and its first harmonics. In the data analyzed, neither tidal nor non-tidal atmospheric models were introduced. Moreover, there are some influences such as multipath which are not able to be modelled in the GNSS data processing and therefore induce a systematic error in the position estimate that appears as correlated noise in the time series (King and Watson 2010).

\section{Noise analysis}

In this section, we address the issues of whether all residual periodicities, as proposed here, need to be taken into consideration when performing noise analysis. All non-modelled geophysical processes or non-included artificial effects in time series lead to an underestimation of errors of velocities, and also to changes in the velocity values themselves. On the other hand, an improper removal of seasonals may result in autocorrelation of residuals. We also must face the fact that there are still some correlations despite subtraction of seasonals, the possible explanation of 
which was given by Agnew and Larsson (2007). They found another important property of GPS system: The time when satellites return to their initial position varies among the satellites of GPS constellation due to singular changes in the mean motion. This in turn means that the draconitic effect in the time series could not be exactly harmonic (Santamaría-Gómez et al. 2011). Other effects related to mismodelling are associated with the Chandler wobble, whose amplitude varies highly in time and its approximation with sine may leave some residual oscillations.

In this study, we subtracted all periodicities for Chandlerian, tropical and draconitic oscillations starting from 1st to 9th harmonic to avoid autocorrelation in the data. These were modelled with the least squares estimation in station-by-station procedure, as described in the previous section. Despite finding the effects of harmonics of a higher order to be insignificant for several stations, we decided to model all of them. We expect this approach to be completely free of any, real or spurious, periods of interest.

The time series of geophysical processes follow the power-law noise character (Agnew 1992; Davis et al. 1994; Langbein and Johnson 1997; Mao et al. 1999; Vasseur and Yodzis 2004). Williams (2003b) investigated power-law dependencies in geodetic time series. He examined the effect of colored noise with non-integer spectral indices $(\kappa)$ and provided equations for rate uncertainties while taking into consideration the noise amplitude $\left(A_{\mathrm{PL}}\right)$, sampling frequency and length of time series. Williams et al. (2004) analyzed time series from 414 permanent stations in nine different solutions for noise content using MLE. The analysis was performed with two noise models. In the first approach, they calculated white noise (WN) only, a combination of WN plus flicker noise (FN), or WN plus random walk (RW). The second approach contained a combination of WN plus power-law (PL). They found that $\mathrm{WN}+\mathrm{FN}$ is clearly the dominant one, and the maximum log-likelihood values confirmed it to be preferential. AmiriSimkooei (2009) analyzed GPS position time series by using the least squares variance component estimation (LSVCE), which gives, in contrast to MLE, unbiased and minimum variance estimators.

In this study, we used the Hector software to undertake the research on time series. All coordinate components were analyzed with pre-assumed power-law plus white noise model, aimed at determining the velocities and their formal errors. Hector uses reformulated MLE with missing data in the observations and maximizes the logarithm of the likelihood function (Bos et al. 2012):

$\ln [p(\mathbf{r})]=-\frac{1}{2}\left[(n-m) \ln 2 \pi+\ln \operatorname{det}(\breve{\mathbf{C}})+\breve{\mathbf{r}}^{T} \breve{\mathbf{C}}^{-1} \mathbf{\mathbf { r }}\right]$

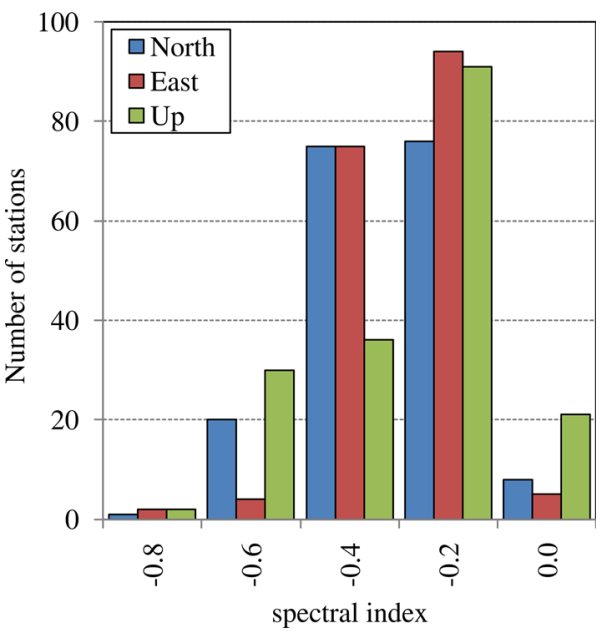

Fig. 6 Histograms of spectral indices of topocentric components

where $n$ is the total number of continuous data, $m$ is the number of missing data, $\mathbf{C}$ is the newly formed covariance matrix of $(n-m) \times(n-m)$ size, and $\mathbf{\mathbf { r }}$ are the residuals.

The parameters that describe different noise models (as, e.g., Equation (6) discussed in Langbein 2004), and thus far the covariance matrix $\breve{C}$ are being changed as far as the greatest value of (5) is found (Bos et al. 2012). In this way, the optimum noise model for the analyzed data is being estimated.

The median percentage of gaps in our time series equalled $0.8 \%$, with a maximum of $12.8 \%$. There were 84 continuous time series out of 180 . Concerning the spectral indices of residuals estimated after substraction of the deterministic part with the method proposed here, it was not noticed that the spectral index of Up component was "less white" than North and East components (Fig. 6). The spectral indices ranged from -0.12 (Up component of BAKO (Cibinong, Indonesia), 20 years of observations) to -0.92 (North component of MATE (Matera, Italy), 22.1 years of observations). We obtained median values of "global" spectral indices equal to: $-0.41 \pm 0.15$, $0.38 \pm 0.12$ and $-0.33 \pm 0.18$ for North, East and Up components, respectively. The shift of spectral indices toward flicker noise, $\kappa=-1$, is probably due to the largescale processes of atmospheric or hydrospheric origin with spatial correlation to some extent.

The thus far commonly used approach of GPS seasonal modelling as the sum of annual and semi-annual tropical curves was adopted here as the reference solution. As was expected, the power-law amplitudes decrease when all periodicities are subtracted from the time series of topocentric components. Moreover, the spectral indices are much "whiter" in comparison with the reference solution (Fig. 7). The largest shift toward white noise was noticed 


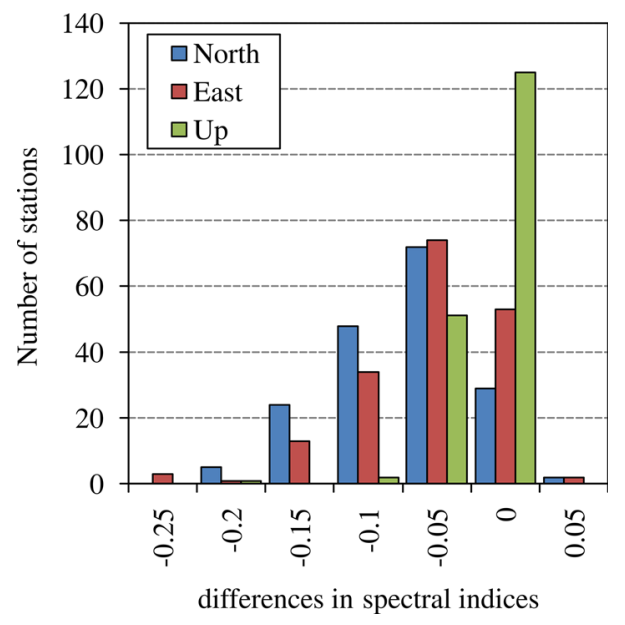

Fig. 7 Histogram of differences in spectral indices between the reference solution and the one proposed here. The negative values mean that residua shifted toward white noise when the proposed subtraction of the seasonal changes was used

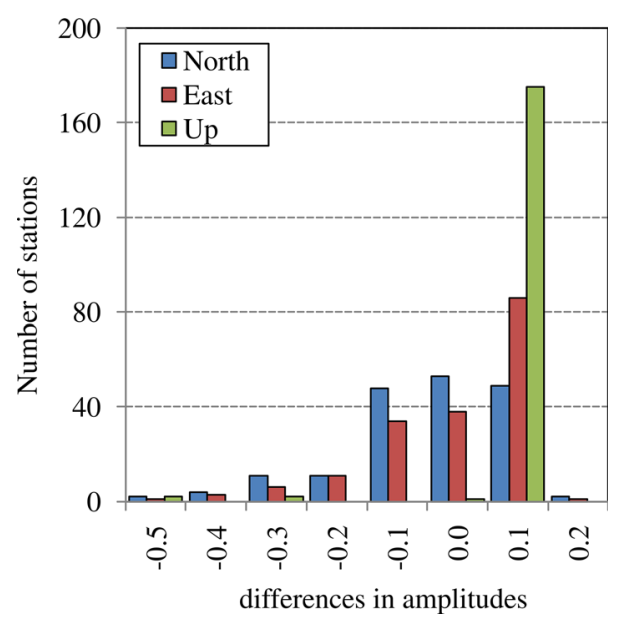

Fig. 8 Histogram of differences in power-law noise amplitudes [mm/ year ${ }^{-\kappa / 4}$ ] between the described and reference approaches. The positive values mean that series residua have a smaller power-law noise amplitude for the proposed approach

for horizontal components, whereas noise character changed for Up only for about $25 \%$ of considered IGS stations.

The power-law noise amplitude decreased between both solutions of $0.7 \mathrm{~mm} / \mathrm{year}^{-\kappa / 4}$ (Fig. 8). The combination of seasonals proposed here resulted in a decrease in noise amplitudes for the vast majority of IGS stations in East and Up. The North component showed odd results on noise amplitudes that were higher after looking at the seasonals in a more complex way. However, the significance of these changes is questionable, since the averaged uncertainty of power-law amplitude in North and East direction is equal to about \pm 0.1 , while in $\mathrm{Up}$ it is $\pm 0.3 \mathrm{~mm} / \mathrm{year}^{-\kappa / 4}$.

The Akaike information criterion (AIC) was used here for a qualitative description of our results. The preferred

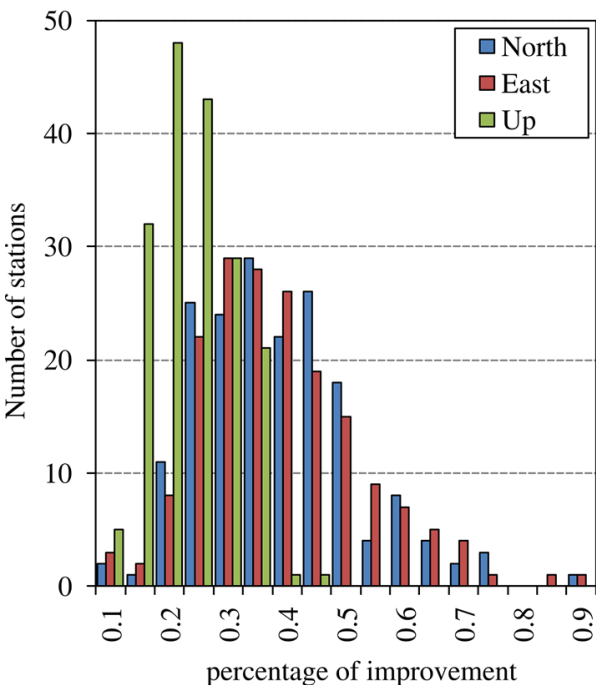

Fig. 9 Comparison of how the proposed solution fits the data based on the AIC values, shown as percentage of improvement. No negative values/worsening have been noticed

model, i.e., the one that minimizes the Kullback-Leibler distance between the model and the truth, is the one with the minimum AIC value. The AIC values were estimated for both seasonal approaches. The proposed assumption of seasonals caused the AIC values to show a decrease in the median value of $30 \%$ (Fig. 9), which in fact means that all the seasonals mentioned here have to be taken into account when analyzing noises.

In order to characterize the geodetically important properties of these two solutions, comparing the reference with the one proposed here, we implemented two types of differences in velocity values determined with linear regression and in the accuracy of the velocity estimated with (Bos et al. 2008):

$m_{v} \approx \pm \sqrt{\frac{A_{\mathrm{PL}}^{2}}{\Delta T^{2-\frac{\kappa}{2}}} \cdot \frac{\Gamma(3-\kappa) \cdot \Gamma(4-\kappa) \cdot(N-1)^{\kappa-3}}{\left[\Gamma\left(2-\frac{\kappa}{2}\right)\right]^{2}}}$

where $N$ is the data length, $\kappa$ means the estimated spectral index, $\Delta T$ is the sampling rate, $A_{P L}$ represents the amplitude of noise, and $\Gamma$ is the gamma function.

The velocities of permanent stations showed a change in values for several of IGS stations when comparing the reference solution with the one proposed. It seems that even the removal of seasonals with a few millimeters of amplitude results in a change in the velocity value (Bogusz and Figurski 2014) of even $0.20 \mathrm{~mm} /$ year. The majority of changes in velocity values were equal to $0.05 \mathrm{~mm} /$ year for the Up component, while the vast majority of velocities remained almost unchanged for North and East (Fig. 10).

Finally, when it comes to the velocity uncertainty, the differences in their values were calculated according to (6) for 180 of analyzed stations. It was noticed that for the majority of stations, the velocity uncertainty decreased 


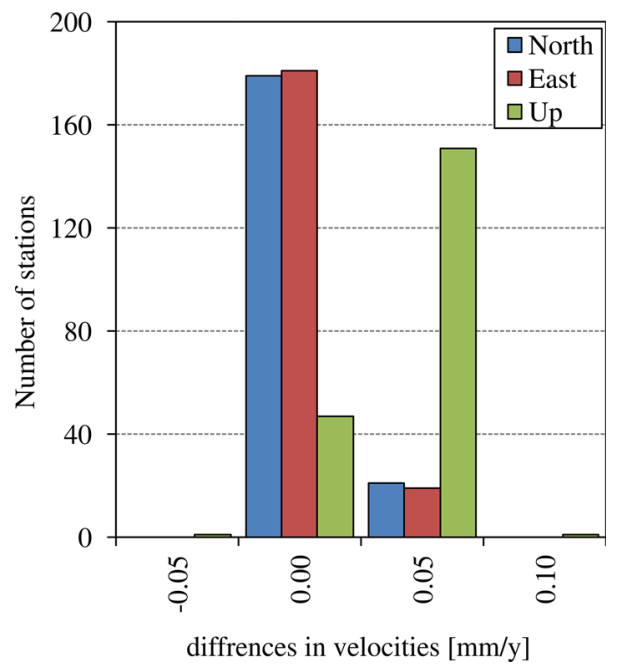

Fig. 10 Histogram of differences in velocity values in ( $\mathrm{mm} / \mathrm{year}$ ) of reference solution that removes the annual and semi-annual tropical periods with the one proposed in this research

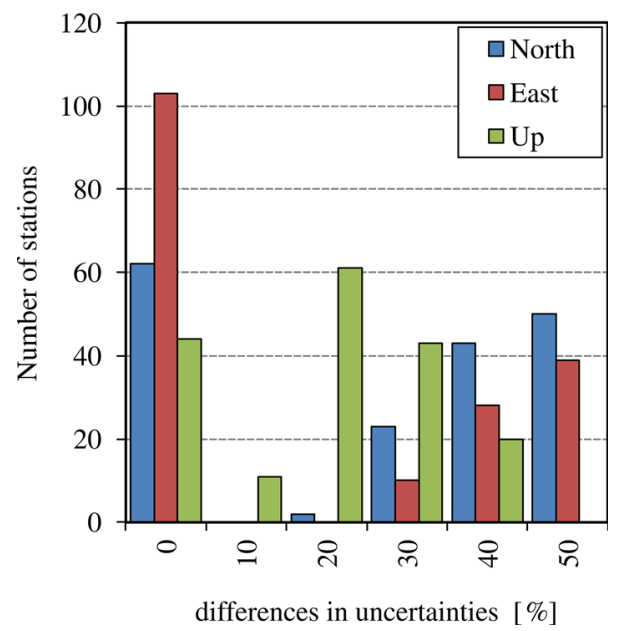

Fig. 11 Histogram of differences in the velocity uncertainties estimates (\%). The positive values mean a decrease in velocity uncertainties after the proposed method

after removal of seasonals as proposed here from -0.03 to $0.10 \mathrm{~mm} /$ year in the most extreme cases. With such long time series, the velocity uncertainties are small, even taking the color noise into consideration, so it is better to use the percentages of those changes. From Fig. 11, we noticed that there are some of the GPS stations that improved their velocity RMS even up to $56 \%$.

\section{Summary and conclusions}

Linear regression is the most common method used today in geophysics to study, e.g., plate tectonic motion. Therefore, the proper interpretation of velocities of permanent GNSS stations in terms of geodynamics requires taking
Table 1 Minimum, maximum and median values of spectral indices and amplitudes of power-law noise obtained

\begin{tabular}{|c|c|c|c|c|c|c|}
\hline & \multicolumn{3}{|c|}{ Spectral index $(\kappa)$} & \multicolumn{3}{|c|}{ Amplitude $\left(A_{\mathrm{PL}}\right)\left[\mathrm{mm} / \mathrm{year}^{-\kappa / 4}\right]$} \\
\hline & MIN & MAX & MED & MIN & MAX & MED \\
\hline North & -0.92 & -0.12 & -0.41 & 0.78 & 3.48 & 1.75 \\
\hline East & -0.83 & -0.14 & -0.37 & 0.86 & 4.17 & 1.55 \\
\hline Up & -0.84 & -0.12 & -0.33 & 3.66 & 8.22 & 4.68 \\
\hline
\end{tabular}

account of the correct deterministic model and the numerous studies on the stochastic character part. It is well known that there is an equivalence between functional and stochastic models (Blewitt 1998) and therefore any unmodelled functional part will show up in the stochastic model and vice versa (Klos et al. 2014b). The removal of all known periodicities causes a significant shift of spectral index toward white noise. Each of the non-subtracted periodicities will naturally cause data to be autocorrelated. Simulations we made prior to this research prove that changing the assumption concerning seasonals from the tropical annual and semi-annual year into Chandler plus tropical annual and its three harmonics, causes a decrease in median spectral indices estimated for IGS stations, processed in network solution mode, from -0.7 up to -0.3 along with a decrease in median amplitude of the powerlaw noise of even $2 \mathrm{~mm} / \mathrm{year}^{-\kappa / 4}$. The assumption of seasonals as a combination of the Chandlerian, tropical and draconitic year with their harmonics up to 9 th, as proposed here, resulted in $100 \%$ of stations to fall into the fractional Gaussian motion $(0<\kappa<-1)$ area. The maximum and median values of spectral indices and power-law noise amplitudes were contrasted in Table 1 . The medians of spectral indices of power-law noises are, respectively, at the level of $-0.41,-0.37$ and -0.33 for North, East and Up with the median amplitudes of $1.75,1.55$ and $4.68 \mathrm{~mm} /$ year ${ }^{-\kappa / 4}$. These results estimated for PPP GPS processing mode are closer to the white noise with spectral index equal to zero and have lower amplitudes than the ones estimated for network solutions (Williams et al. 2004; Teferle et al. 2008; Klos et al. 2014a).

Moreover, in contrast to other researches (Teferle et al. 2008; Williams et al. 2004) it was not noticed that the vertical magnitudes are larger than the horizontal ones. The ratio of spectral indices in the vertical and horizontal direction was calculated as:

$r=\frac{\kappa_{\mathrm{U}}}{\frac{1}{2}\left(\kappa_{\mathrm{N}}+\kappa_{\mathrm{E}}\right)}$

with $\kappa_{N}, \kappa_{E}$ and $\kappa_{U}$ being spectral indices of North, East and Up, respectively. The results show that a minimum ratio of $r=0.30$, a maximum ratio of $r=2.11$ and a median of $r=0.92$. Of analyzed stations, $56.1 \%$ had a 
ratio of $r<1$, which means that the spectral indices for $\mathrm{Up}$ are closer to zero and so closer to white noise at the same time than for North and East components.

The AIC criterion was estimated here for two seasonal approaches. The reference solution being the sum of tropical annual and semi-annual periods showed worse AIC characteristic than the new approach proposed here being the sum of Chandlerian, tropical and draconitic curves up to their 9th harmonics. These three conclusions show the correctness of our approach.

The source of noises in GPS-derived time series may be a combination of global, regional and local site-dependent effects. The regional effects named common-mode errors that influence the whole network of stations may be removed from the data with a range of spatiotemporal filtering methods. This problem has already been raised by several authors (Williams et al. 2004; Amiri-Simkooei 2013; Bogusz et al. 2015). This kind of filtering that takes into account other noise sources than mis- or unmodelled seasonals would improve the noise results in a similar way as our approach did.

Acknowledgments This research was financed by the Polish National Science Centre, Grant No. UMO-2014/15/B/ST10/03850. JPL time series were accessed from ftp://sideshow.jpl.nasa.gov/pub/ JPL_GPS_Timeser/repro2011b/raw. The maps were drawn in the Generic Mapping Tools. M. Bos and S. Williams for their insightful comments and efficient discussion are gratefully acknowledged.

Open Access This article is distributed under the terms of the Creative Commons Attribution 4.0 International License (http://crea tivecommons.org/licenses/by/4.0/), which permits unrestricted use, distribution, and reproduction in any medium, provided you give appropriate credit to the original author(s) and the source, provide a link to the Creative Commons license, and indicate if changes were made.

\section{References}

Agnew D (1992) The time-domain behavior of power-law noises. Geophys Res Lett 19(4):333-336

Agnew DC, Larson KM (2007) Finding the repeat times of the GPS constellation. GPS Solut 11(1):71-76. doi:10.1007/s10291-0060038-4

Amiri-Simkooei AR (2009) Noise in multivariate GPS position time series. J Geod 83(2):175-187. doi:10.1007/s00190-008-0251-8

Amiri-Simkooei AR (2013) On the nature of GPS draconitic year periodic pattern in multivariate position time series. J Geophys Res Solid Earth 118(5):2500-2511. doi:10.1002/jgrb.50199

Amiri-Simkooei AR, Tiberius CCJM, Teunissen PJG (2007) Assessment of noise in GPS coordinate time series: methodology and results. J Geophys Res 112(B7):B07413. doi:10.1029/2006JB004913

Blewitt G (1998) GPS data processing methodology: from theory to application. In: Teunissen PG, Kleuberg A (eds) GPS for Geodesy. Springer, Berlin, pp 231-270

Blewitt G, Lavallée D (2002) Effect of annual signals on geodetic velocity. J Geophys Res 107(B7):2145. doi:10.1029/ 2001JB000570
Bogusz J, Figurski M (2012) GPS-derived height changes in diurnal and sub-diurnal timescales. Acta Geophys 60(2):295-317. doi:10.2478/s11600-011-0074-5

Bogusz J, Figurski M (2014) Annual signals observed in regional GPS networks. Acta Geodyn Geomater 11(174):125-131. doi:10. 13168/AGG.2014.0003

Bogusz J, Figurski M, Gruszczynki M, Klos A (2015) Spatiotemporal filtering for determination of common mode error in regional GNSS networks. Open Geosci 7(1):140-148. doi:10. 1515/geo-2015-0021

Bos MS, Fernandes RMS, Williams SDP, Bastos L (2008) Fast error analysis of continuous GPS observations. J Geod 82(3):157-166. doi:10.1007/s00190-007-0165-x

Bos MS, Bastos L, Fernandes RMS (2010) The influence of seasonal signals on the estimation of the tectonic motion in short continuous GPS time-series. J Geodyn 49(4):205-209. doi:10. 1016/j.jog.2009.10.005

Bos MS, Fernandes RMS, Williams SDP, Bastos L (2012) Fast error analysis of continuous GNSS observations with missing data. J Geod 87(4):351-360. doi:10.1007/s00190-012-0605-0

Bruni S, Zerbini S, Raicich F, Errico M, Santi E (2014) Detecting discontinuities in GNSS coordinate time series with STARS: case study, the Bologna and Medicina GPS sites. J Geod 88(12):1203-1214. doi:10.1007/s00190-014-0754-4

Collilieux X, Altamimi Z, Coulot D, Ray J, Sillard P (2007) Comparison of very long baseline interferometry, GPS, and satellite laser ranging height residuals from ITRF2005 using spectral and correlation methods. J Geophys Res 112:B12403. doi: 10.1029/2007JB004933

Davis A, Marshak A, Wiscombe A, Cahalan R (1994) Multifractal characterizations of nonstationarity and intermittency in geophysical fields: observed, retrieved or simulated. J Geophys Res 99(D4):8055-8072

Davis JL, Wernicke BP, Tamisiea ME (2012) On seasonal signals in geodetic time series. J Geophys Res 117(B1):B01403. doi:10. 1029/2011JB008690

Desai S (2005) Observing the pole tide with satellite altimetry. J Geophys Res 107(C11):C113186. doi:10.1029/2001JC001224

Dong D, Fang P, Bock Y, Cheng MK, Miyazaki S (2002) Anatomy of apparent seasonal variations from GPS-derived site position time series. J Geophys Res Solid Earth. doi:10.1029/2001JB000573

Gazeaux J, Williams S, King M, Bos M, Dach R, Deo M, Moore AW, Ostini L, Petrie E, Roggero M, Teferle FN, Olivares G, Webb FH (2013) Detecting offsets in GPS time series: first results from the detection of offsets in GPS experiment. J Geophys Res Solid Earth 118(5):2397-2407. doi:10.1002/jgrb.50152

Godin G (1972) The analysis of tides. University of Toronto Press, Toronto, p 264

Griffiths J, Ray JR (2013) Sub-daily alias and draconitic errors in the IGS orbits. GPS Solut 17(3):413-422. doi:10.1007/s10291-0120289-1

Gross R, Blewitt G, Clarke PJ, Lavallée D (2004) Degree-2 harmonics of the Earth's mass load estimated from GPS and Earth rotation data. Geophys Res Lett 31:L07601. doi:10.1029/ 2004GL019589

King MA, Watson CS (2010) Long GPS coordinate time series: multipath and geometry effects. J Geophys Res 115(B4):B04403. doi:10.1029/2009JB006543

King MA, Watson CS, Penna NT, Clarke PJ (2008) Subdaily signals in GPS observations and their effect at semiannual and annual periods. Geophys Res Lett 35(3):L03302. doi:10.1029/2007GL032252

Klos A, Bogusz J, Figurski M, Kosek W (2014a) Uncertainties of geodetic velocities from permanent GPS observations: Sudeten case study. Acta Geodyn Geomater 11(175):125-131. doi:10. 13168/AGG.2014.0003 
Klos A, Bogusz J, Figurski M, Kosek W (2014b) Irregular variations in the GPS time series by the probability and noise analysis. Surv Rev 47(342):163-173. doi:10.1179/1752270614Y.0000000133

Kouba J (2005) Comparison of polar motion with oceanic and atmospheric angular momentum time series for 2-day to Chandler periods. J Geod 79(1-3):33-42. doi:10.1007/s00190-005-0440-7

Langbein J (2004) Noise in two-color electronic distance meter measurements revisited. J Geophys Res. doi:10.1029/ 2003JB002819

Langbein JO, Johnson H (1997) Correlated errors in geodetic time series: implications for time-dependent deformation. J Geophys Res 102(B1):591-603

Lomb NR (1976) Least-squares frequency analysis of unequally spaced data. Astrophys Space Sci 39(2):447-462. doi:10.1007/ BF00648343

Mao A, Harrison CGA, Dixon TH (1999) Noise in GPS coordinate time series. J Geophys Res 104(B2):2797-2816

Melchior P (1983) The tides of the planet earth, 2nd edn. Pergamon Press, Oxford

Penna NT, Stewart MP (2003) Aliased tidal signatures in continuous GPS height time series. Geophys Res Lett 30(23):2184. doi:10. 1029/2003GL018828

Penna NT, King MA, Stewart MP (2007) GPS height time series: short-period origins of spurious long-period signals. J Geophys Res 112(B2):B02402. doi:10.1029/2005JB004047

Ray J, Altamimi Z, Collilieux X, van Dam T (2008) Anomalous harmonics in the spectra of GPS position estimates. GPS Solut 12(1):55-64. doi:10.1007/s10291-007-0067-7

Rodionov S, Overland JE (2005) Application of a sequential regime shift detection method to the Bering Sea ecosystem. ICES J Mar Sci 62:328-332. doi:10.1016/j.icesjms.2005.01.013

Rodriguez-Solano CJ, Hugentobler U, Steigenberger P, Lutz S (2012) Impact of earth radiation pressure on GPS position estimates. J Geod 86(5):309-317. doi:10.1007/s00190-011-0517-4

Santamaría-Gómez A, Bouin MN, Collilieux X, Woppelmann G (2011) Correlated errors in GPS position time series: implications for velocity estimates. J Geophys Res 116(B1):B01405. doi:10.1029/2010JB007701

Scargle JD (1982) Studies in astronomical time series analysis. IIStatistical aspects of spectral analysis of unevenly spaced data. Astrophys J 263:835-853. doi:10.1086/160554

Teferle FN, Williams SDP, Kierulf HP, Bingley RM, Plag HP (2008) A continuous GPS coordinate time series analysis strategy for high accuracy vertical land movements. Phys Chem Earth 33(3-4):205-216. doi:10.1016/j.pce.2006.11.002

Tregoning P, Watson C (2009) Atmospheric effects and spurious signals in GPS analyses. J Geophys Res 114(B9):B09403. doi:10.1029/2009JB006344

van Dam TM, Blewitt G, Heflin MB (1994) Atmospheric pressure loading effects on the global positioning system coordinate determinations. J Geophys Res 99(B12):23939-23950

Vasseur DA, Yodzis P (2004) The color of environmental noise. Ecology 85(4):1146-1152. doi:10.1890/02-3122
Williams SDP (2003a) Offsets in global positioning system time series. J Geophys Res 108(B6):2310. doi:10.1029/ 2002JB002156

Williams SDP (2003b) The effect of coloured noise on the uncertainties of rates estimated from geodetic time series. J Geod 76(9-10):483-494. doi:10.1007/s00190-002-0283-4

Williams SDP, Bock Y, Fang P, Jamason P, Nikolaidis RM, Prawirodirdjo L, Miller M, Johnson D (2004) Error analysis of continuous GPS position time series. J Geophys Res 109(B3):B03412. doi:10.1029/2003JB002741

Wu X, Heflin MB, Ivins ER, Fukumori I (2006) Seasonal and interannual global surface mass variations from multisatellite geodetic data. J Geophys Res 111(B9):B09401. doi:10.1029/ 2005JB00410

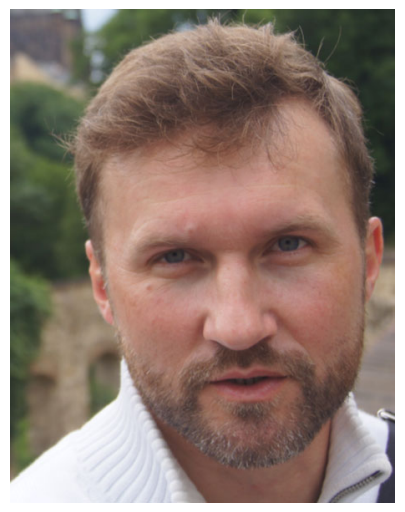

Janusz Bogusz made his $\mathrm{PhD}$ thesis related to geodynamics and atmospheric tides in 2000 . His habilitation thesis (2012) concerned applicability of shorttime GPS solutions to the studies of residual dynamic deformational changes in tidal frequencies. Presently, he works as the associate professor at the Military University of Technology in Warsaw.

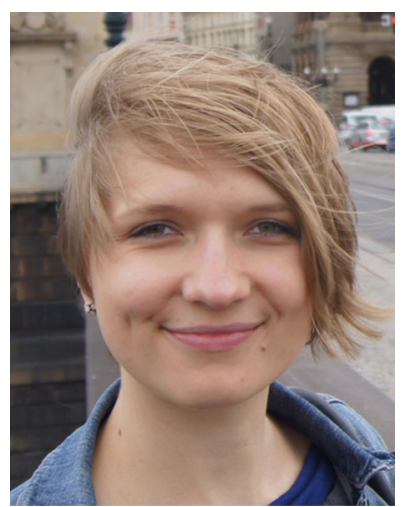

Anna Klos graduated from the Military University of Technology in 2012. Her Master thesis dealt with evaluation of shortterm stability of permanent stations of ASG-EUPOS network. Now, she is a PhD student at the Military University of Technology in Warsaw. Her research concerns the time series analysis with a main focus on noise analysis. 